
months

\title{
Stagnell $\mathrm{S}^{1}$, Koller $\mathrm{M}^{2}$, Steyer $\mathrm{E}^{2}$, Theisen $\mathrm{K}^{2}$, Jakse $\mathrm{N}^{1}$ \& Payer $\mathrm{M}^{1}$
}

${ }^{1}$ Department of Oral Surgery and Orthodontics, School of Dentistry, Medical University Graz, Graz, Austria 2 Department of Restorative Dentistry, Periodontology and Prosthodontics, School of Dentistry, Medical University Graz, Graz, Austria

\section{Abstract}

\section{Background}

In spite of lacking evidence for advantages of substitutes over titanium, it seems that there is a basic movement of patients and dentists toward the use of metal free solutions also in implant dentistry. This tendency is strongly supported by of a growing cohort of patients with concerns over titanium sensitivity, allergies or intolerances irrespective of only weak evidence for clinical relevance as well as clinical concerns regarding aesthetic outcomes in the anterior region.

\section{Aim/Hypothesis}

The aim of this study was to evaluate the outcomes of allceramic crowns on two-piece zirconia implants in a prospective randomized controlled study after up to 80 months

\section{Materials and Methods}

21 patients with a total of 28 implants (14 zirconia and 14 titanium) were reviewed up to 80 months following restoration. Bleeding on probing (BOP), plaque index (PI), pink aesthetic score (PES), mean radiographic marginal bone resorption, and score (PES), man radiographic
implant survival was determined.

\section{Results}

After a mean observation time of 80.9 months $(S D \pm 5.5)$ the following data were assessed: Mean marginal bone level of $-1.38 \mathrm{~mm}$ at zirconia $(\mathrm{SD} \pm 0.81)$ and $-1.17 \mathrm{~mm}$ at titanium implants (SD \pm 0.73$)$. BOP of $16.43 \%(S D \pm 6.16)$ at zirconia and $12.60 \%$ (SD \pm 7.66$)$ at titanium implants. Pl of $11.07 \%$ (SD $\pm 8.11)$ at zirconia and $15.20 \%(S D \pm 15.58)$ at titanium implants. PES of $11.38(\mathrm{SD} \pm 0.92)$ at zirconia and $11.56(\mathrm{SD} \pm$ 1.01) at titanium implants respectively. Two zirconia implants 1.01) at titanium implants respectively. Two zirconia implants and 1 titanium were lost within the evaluated period after
implant surgery resulting in a survival rate of $85.7 \%$ for zirconia and $93.3 \%$ for titanium implants.

\section{Conclusions and Clinical Implications}

In the present pilot trial no significant differences between adhesively luted zirconia abutments and all-ceramic restorations on two-piece zirconia implants could be observed after up to 80 months compared to a titanium control group. The small study population needs to be taken in consideration when interpreting the results.

\section{Background and Aim}

In spite of lacking evidence for advantages of substitutes over titanium, it seems that there is a movement of patients and dentists toward the use of metal free solutions also in implant dentistry, supported by a growing cohort of patients with concerns over titanium sensitivity, allergies or intolerances irrespective of only weak evidence for clinical relevance [1-4].

So far most of the studies on zirconia implants have reported on the outcomes of single-piece implants as the connection using a regular screw type connection between ceramic implants and abutments has seemed to be a technical challenge [5]

In this presented clinical trial a two-piece zirconia implant system was used, as previously described in the paper by Payer et al. [6], in which the abutments had to be cemented adhesively onto the implants (Fig. 1).

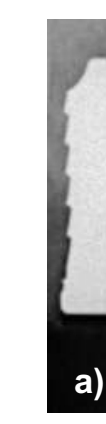

\section{Methods and Materials}

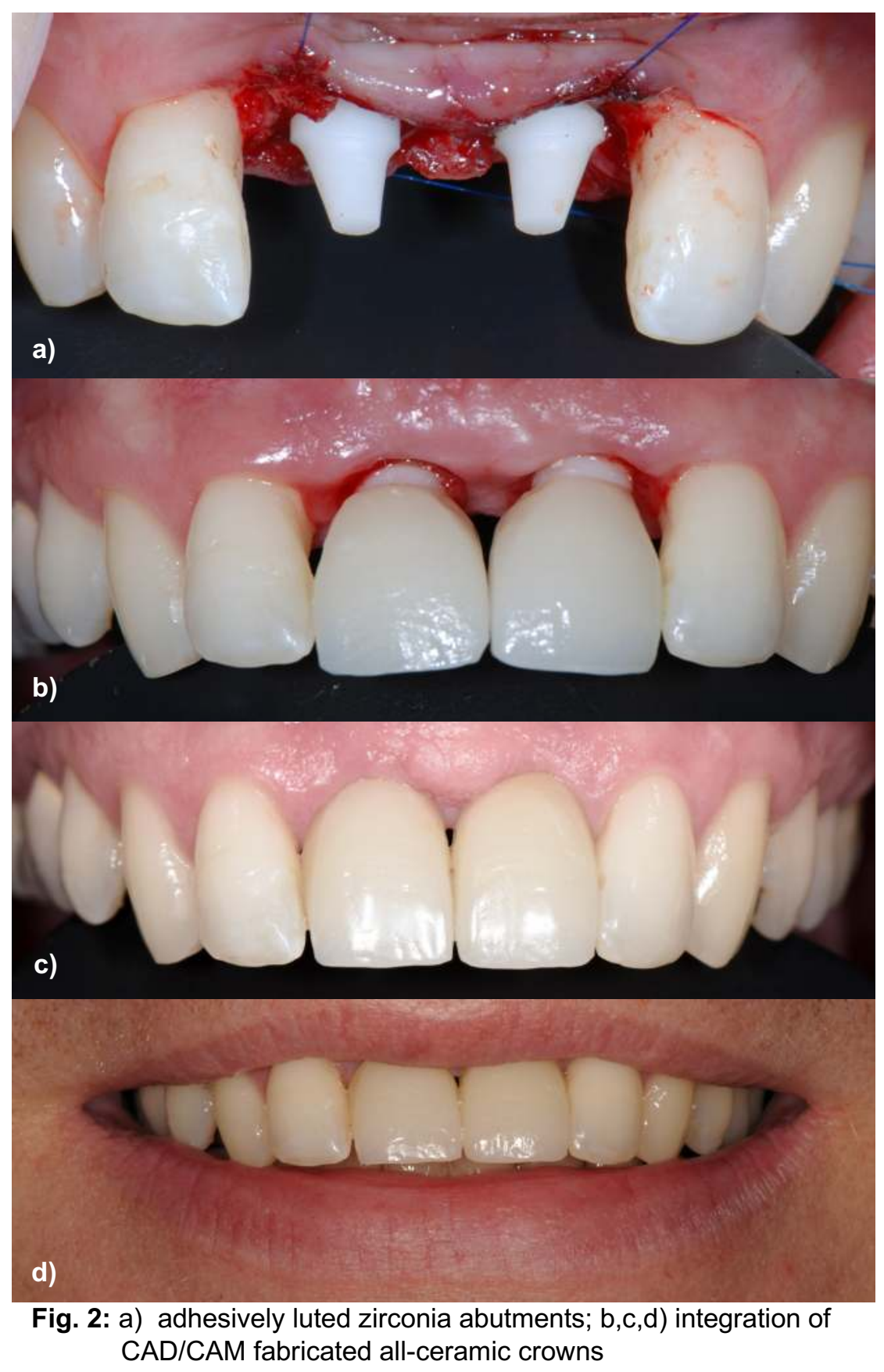

The original study was conducted between 2009 and 2010 as a prospective, randomized and controlled clinical single-centre pilot trial at the Dental School, Medical University of Graz, Austria.

Only patients matching the inclusion criteria, e.g. sufficient amount of horizontal and vertical bone and soft tissue volume for the placement of implants with a minimum length of $10 \mathrm{~mm}$ and a width of $4 \mathrm{~mm}$, were consecutively included in the investigation: All implants were placed in positions with corresponding and opposing natural teeth in occlusion. Second-stage surgery was carried out after 4 months for mandibular and 6 months for maxillary implants. Prefabricated standard ceramic abutments were luted adhesively into the zirconia implants after rubber dam application, whereas screw retained titanium abutments were used for the titanium implants. Conventional impressions with polyether were carried out for fabrication of all-ceramic lithium disilicate crowns (Fig. 2).

For measuring the marginal bone level standardised $x$-rays were made. Implant diameter was defined as reference. Clinical examinations were done to asses the outcome of BOP and PI. The PES was evaluated by an experienced dentist using digital photographs (Fig. 3).

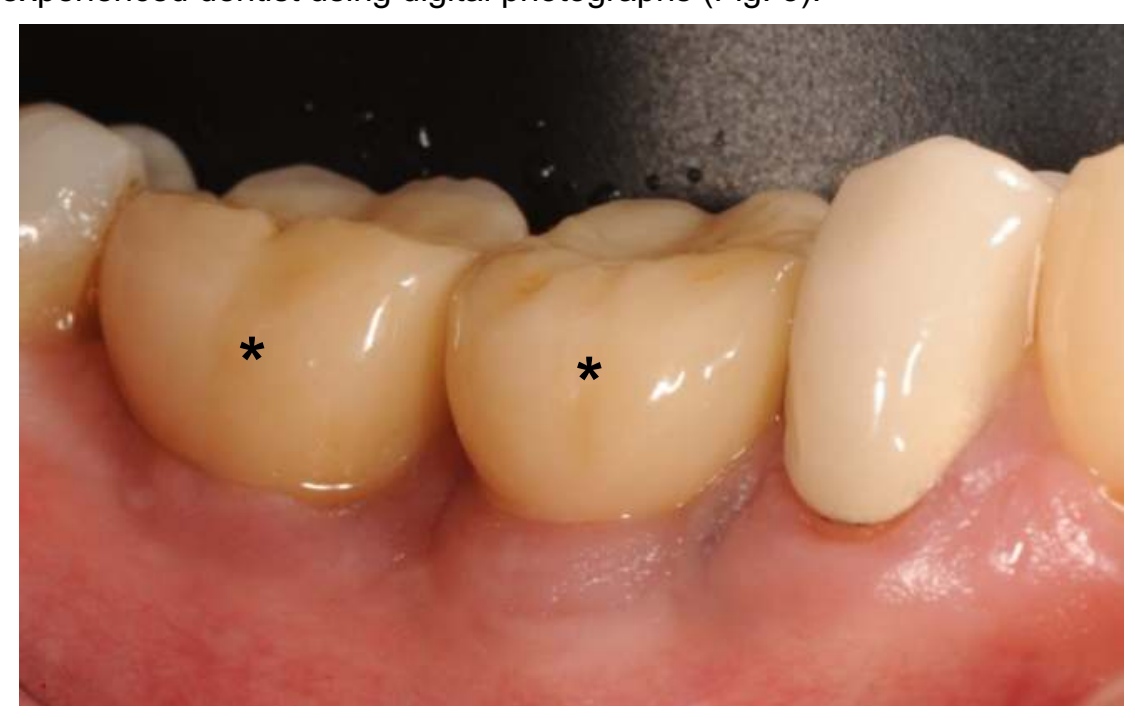

Fig. 3: soft-tissue around titanium implants (implant sites marked by *)

\section{Results}

Of the initial 31 study implants (16 zirconia and 15 titanium implants) in 22 healthy patients, 28 implants inserted in 21 healthy patients could be evaluated after 80 months. 8 months and 25 months after restoration two zirconia implants were lost. Eighty months post restoration one titanium implant had to be removed due to periimplantitis. All other implants showed stable results. As such, 14 zirconia and 14 titanium implants were included in the follow-up examination - resulting in a survival of 14 out of 16 zirconia and 14 out of 15 titanium implants. At the follow up after 80 months mean marginal bone level at zirconia revealed $-1.38 \mathrm{~mm}(S D \pm 0.81)$ and $1.17 \mathrm{~mm}$ at titanium implants ( $\mathrm{SD} \pm 0.73$ ) (Fig. 4). A BOP of $16.43 \%$ $(\mathrm{SD} \pm 6.16)$ at zirconia and $12.60 \%(\mathrm{SD} \pm 7.66)$ at titanium implants was assessed (Fig. 5). PI showed 11.07\% (SD \pm 8.11 ) at zirconia and $15.20 \%$ (SD \pm 15.58 ) at titanium implants (Fig. 6). A PES of 11.38 $(\mathrm{SD} \pm 0.92)$ at zirconia and $11.56(\mathrm{SD} \pm 1.01)$ at titanium implants respectively was detected (Fig. 7).

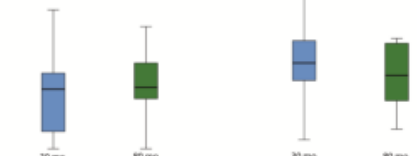

Fig. 4: Mean marginal bone leve

Fig. 5: BOP

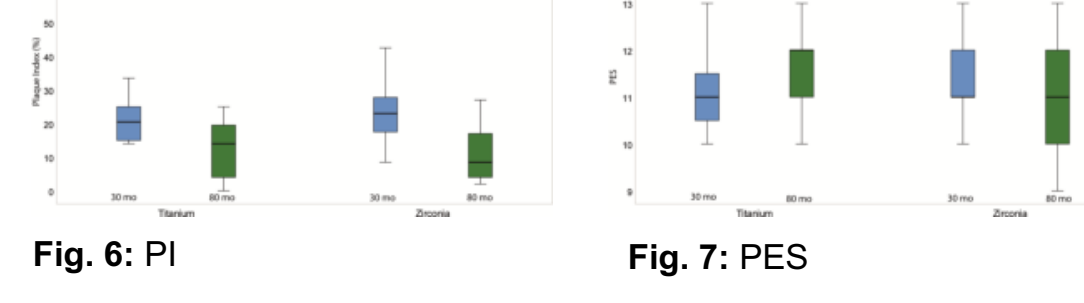

\section{Conclusion}

Within the current evidence-base, there is little if no other RCT examining the outcomes of zirconia implants in comparison to conventional titanium of identical shape. The data presented here highlight favourable outcomes when considering zirconia implants as a viable metal-free alternative within implantology. Of course, in comparison to the long-standing history of titanium, more work is required in the field of zirconia implants: Developments are needed with respect to prosthetic solutions for zirconia implants as actually it relies greatly on treatment planning and a high level of clinical skills to execute precisely.

\section{References}

1 Sivaraman, K., Chopra, A., Narayan, A.I. \& Balakrishnan, D. (2018) Is zirconia a viable alternative to titanium for oral implant? A critical review. Journal of prosthodontic research 62 2 Fage, S.W., Muris, J., Jakobsen, S.S. \& Thyssen, J.P. (2016) Titanium: a review on
exposure, release, penetration, allergy, epidemiology, and clinical reactivity. Contact dermatitis 74: 323-345. 3 Hosolk M, 323.345. 60: 213-219.
4 Thomas, P., Iglhaut, G., Wollenberg, A., Cadosch, D. \& Summer, B. (2013) Allergy or tolerance: reduced inflammatory cytokine response and concomitant IL-10 production of
lymphocytes and monocytes in symptom-free titanium dental implant patients. BioMed
research international 2013: 539834 . 5 Preis, V., Kammermeier, A., Handel, G. \& Rosentritt, M. (2016) In vitro performance of two-
piece zirconia implant systems for anterior application. Dental materials : official publication of the Academy of Dental Materials 32: 765-774.
6 Payer, M., Heschl, A., Koller, M., Arnetzl, G., Lorenzoni, M. \& Jakse, N. (2015) All-ceramic restoration of zirconia two-piec
implants research 26: $371-376$. 